\title{
SpringerBriefs in Mathematical Physics
}

\author{
Volume 9
}

\section{Series editors}

Nathanaël Berestycki, Cambridge, UK

Mihalis Dafermos, Princeton, USA

Tohru Eguchi, Tokyo, Japan

Atsuo Kuniba, Tokyo, Japan

Matilde Marcolli, Pasadena, USA

Bruno Nachtergaele, Davis, USA 
More information about this series at http://www.springer.com/series/11953 
Wim Beenakker - Thijs van den Broek Walter D. van Suijlekom

\section{Supersymmetry and Noncommutative Geometry}

黛 Springer 
Wim Beenakker

Faculty of Science

Radboud University Nijmegen

Nijmegen

The Netherlands

and

University of Amsterdam

Amsterdam

The Netherlands
Thijs van den Broek

Nikhef

Radboud University Nijmegen

Nijmegen

The Netherlands

Walter D. van Suijlekom

Faculty of Science

Radboud University Nijmegen

Nijmegen

The Netherlands

ISSN 2197-1757

ISSN 2197-1765 (electronic)

SpringerBriefs in Mathematical Physics

ISBN 978-3-319-24796-0

ISBN 978-3-319-24798-4 (eBook)

DOI 10.1007/978-3-319-24798-4

Library of Congress Control Number: 2015950189

Springer Cham Heidelberg New York Dordrecht London

(C) The Author(s) 2016

This work is subject to copyright. All rights are reserved by the Publisher, whether the whole or part of the material is concerned, specifically the rights of translation, reprinting, reuse of illustrations, recitation, broadcasting, reproduction on microfilms or in any other physical way, and transmission or information storage and retrieval, electronic adaptation, computer software, or by similar or dissimilar methodology now known or hereafter developed.

The use of general descriptive names, registered names, trademarks, service marks, etc. in this publication does not imply, even in the absence of a specific statement, that such names are exempt from the relevant protective laws and regulations and therefore free for general use.

The publisher, the authors and the editors are safe to assume that the advice and information in this book are believed to be true and accurate at the date of publication. Neither the publisher nor the authors or the editors give a warranty, express or implied, with respect to the material contained herein or for any errors or omissions that may have been made.

Printed on acid-free paper

Springer International Publishing AG Switzerland is part of Springer Science+Business Media (www.springer.com) 


\section{Preface}

The Standard Model of particle physics is an extremely successful theory, describing all currently known elementary particles and their nongravitational interactions. Experimentally, it is tested with previously unencountered precision. However, at the same time it is also clear that at some point it will meet its bounds. For instance, the gravitational force is not included, there are large quadratic corrections to the Higgs mass and it does not account for dark matter. We are therefore in need of a new theory, respecting the various constraints from both experiment and theory, from which the Standard Model emerges as a low-energy limit.

The Standard Model can be beautifully derived from geometrical principles using noncommutative geometry [1,2]. This includes the Higgs field and the Higgs mechanism. Moreover, the Higgs mass could be predicted in this geometrical framework, but its value turned out to be off (see Sect. 1.2.3 below). At the same time, any prediction of this sort depends on the contents of the chosen noncommutative manifold (e.g. [3]). Application of noncommutative geometry thus gives us new ways to understand the structure of gauge theories in general and the Standard Model in particular. The question is whether it, in addition, can teach us more about reality - via the correct prediction or retrodiction of particle massesthan ordinary field theory does. In particular, the hope is that there is a theory that can be considered an extension of the noncommutative Standard Model and that, on top of being phenomenologically viable, yields a sufficiently lower value for the Higgs mass.

The minimally supersymmetric Standard Model (MSSM, Sect. 1.1.1) is a particularly prominent example of physics beyond the Standard Model. Although the question whether supersymmetry is a real symmetry of nature is still open, the merits of the MSSM and models akin alone make them worthwhile to analyze in full detail.

This is the main motivation to search for a theory from noncommutative geometry that describes the MSSM (or something alike), which is the main subject of this book.

To achieve this aim, we will first study the more general question if the spectral action (cf. Eq. 1.21 below) that stems from noncommutative geometry can exhibit 
supersymmetry. We do this in Chap. 2. If one is after phenomenologically viable theories of supersymmetry, the question on how to break it again is unavoidable. We therefore turn to this matter in Chap. 3. Finally, we apply the framework developed in Chap. 2 to the almost-commutative geometry that is to give the MSSM in this context in Chap. 4.

Previous attempts to reconcile supersymmetry with noncommutative geometry have been made, see e.g., [4-7], but have not led to conclusive answers. We distinguish ourselves from these approaches in the following ways:

- We try to stay as close as possible to the framework of noncommutative geometry, not digressing into superspace and superfields and the likes.

- All attempts were made prior to the introduction of the spectral action (Eq. 1.21).

Since the latter has proven itself so well in obtaining the Standard Model and since the (predictive) power of the noncommutative method relies heavily on it, we choose it to be our action functional and will ask ourselves in Chap. 2 the question "for what noncommutative geometries is the action supersymmetric?" or "what are supersymmetric noncommutative geometries?" This is in contrast to the question "what actions are supersymmetric?" that one typically tries to answer using the superfield formalism. Note the crucial difference here; the intimate connection between an almost-commutative geometry and its associated action forbids us to manually add terms to the latter.

Concerning the prerequisites for reading this book, we assume familiarity with the basic notions in high energy physics (such as action functionals, Lorentz invariance, gauge symmetries) referring to the standard textbooks such as [8-10]. For the two central themes of this book (noncommutative geometry and supersymmetry), references for further reading are included in the main text.

\section{Acknowledgments}

We thank Alain Connes, Gianni Landi, Matilde Marcolli and Aldo Rampioni from Springer for their editorial support for transforming this material into the present book form.

We would like to thank John Barrett for useful comments. Van den Broek would like to thank the Dutch Foundation for Fundamental Research on Matter (FOM) for funding this work.

Nijmegen

Wim Beenakker Thijs van den Broek Walter D. van Suijlekom 


\section{References}

1. A. Connes, Noncommutative Geometry (Academic Press, San Diego, 1994)

2. A. Chamseddine, A. Connes, M. Marcolli, Gravity and the standard model with neutrino mixing. Adv. Theor. Math. Phys. 11, 991-1089 (2007)

3. A. Chamseddine, A. Connes, Resilience of the Spectral Standard Model. J. High Energy Phys. 1209, $104(2012)$

4. F. Hussain, G. Thompson, Noncommutative geometry and supersymmetry. Phys. Lett. B 260, 359-364 (1991)

5. F. Hussain, G. Thompson, Noncommutative geometry and supersymmetry 2. Phys. Lett. B 265, 307-310 (1991)

6. W. Kalau, M. Walze, Supersymmetry and noncommutative geometry. J. Geom. Phys. 22, 77 102 (1997)

7. J. Frohlich, O. Grandjean, A. Recknagel, Supersymmetric quantum theory and noncommutative geometry. Commun. Math. Phys. 203, 119 (1999)

8. M. Peskin, D. Schroeder, An Introduction to Quantum Field Theory (Westview Press, Boulder, 1995)

9. S. Weinberg, The Quantum Theory of Fields, Volume 2 (Cambridge University Press, Cambridge, 2005)

10. W. Cottingham, D. Greenwood, An Introduction to the Standard Model of Particle Physics (Cambridge University Press, Cambridge, 2007) 


\section{Contents}

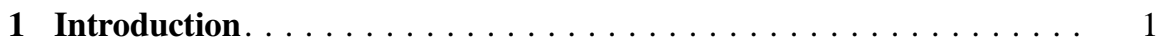

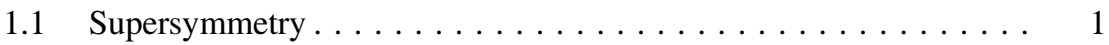

1.1.1 The Supersymmetric Version of the Standard Model. ... . 4

1.2 Noncommutative Geometry . . . . . . . . . . . . . . . . . 5

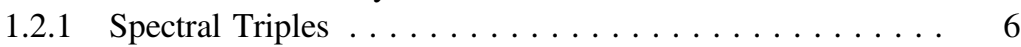

1.2.2 Gauge Fields and the Action Functional . . . . . . . . 10

1.2.3 The Noncommutative Standard Model (NCSM) . . . . . . . 13

1.2.4 Finite Spectral Triples and Krajewski Diagrams . . . . . . . 15

References ......................... 20

2 Supersymmetric Almost-Commutative Geometries . . . . . . . . 23

2.1 Noncommutative Geometry and R-Parity . . . . . . . . . . 23

2.2 Supersymmetric Spectral Triples . . . . . . . . . . . . . . . 24

2.2.1 First Building Block: The Adjoint Representation..... . . 25

2.2.2 Second Building Block: Adding Non-adjoint Representations. . . . . . . . . . . . . . . . . 29

2.2.3 Third Building Block: Extra Interactions . . . . . . . . 46

2.2.4 Higher Degree Building Blocks?. . . . . . . . . . . . 57

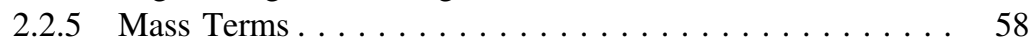

2.3 Conditions for a Supersymmetric Spectral Action ... . . . . . 67

2.3.1 Applied to a Single Building Block of the Third Type ... 73

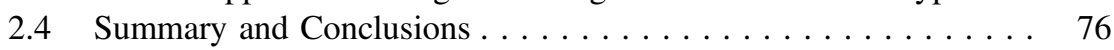

References ........................ 105

3 Supersymmetry Breaking . . . . . . . . . . . . . . . . . . 107

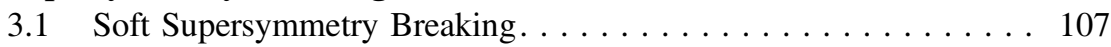

3.2 Soft Supersymmetry Breaking Terms from

the Spectral Action . . . . . . . . . . . . . . . . . 108

3.2.1 Scalar Masses (E.g. Higgs Masses) . . . . . . . . . . . . 109

3.2 .2 Gaugino Masses . . . . . . . . . . . . . . . . . . 110

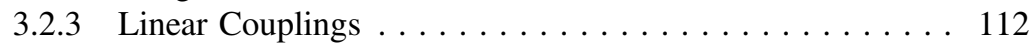

3.2 .4 Bilinear Couplings . . . . . . . . . . . . . . . . 113 
3.2.5 Trilinear Couplings . . . . . . . . . . . . . . . . . 114

3.3 Summary and Conclusions . . . . . . . . . . . . . . 116

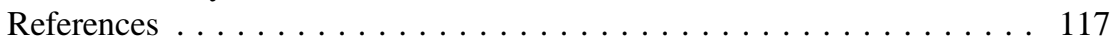

4 The Noncommutative Supersymmetric Standard Model . . . . . . . . . . 119

4.1 Obstructions for a Supersymmetric Theory . . . . . . . . . . . . . . . . 119

4.2 The Building Blocks of the MSSM . . . . . . . . . . . . . . 120

4.3 Identification of Particles and Sparticles . . . . . . . . . . . . . 126

4.3.1 The Gauge Group and Hypercharges. . . . . . . . . . . . 126

4.3.2 Unimodularity in the MSSM . . . . . . . . . . . . . . . . . . . . . . . . 128

4.4 Supersymmetry of the Action . . . . . . . . . . . . . . . . . . . . . . . . . . 132

4.5 Summary and Conclusions . . . . . . . . . . . . . . . 136

References ........................... 137 